\section{A new spectrum of personality disorders?}

Schug et al's article on psychophysiological and behavioural characteristics of individuals with comorbid antisocial personality disorder (ASPD) and schizophrenia-spectrum personality disorder (SSPD) opens the possibility of the existence of a new spectrum of personality disorders. But reading the article with care raises some questions.

First, the community-based study population which was recruited from temporary employment agencies appears to have a prevalence of personality disorder of the order of what we would observe in psychiatric in-patient units. ${ }^{2}$ The total prevalence in this study population was $52.4 \%$, which is way beyond the prevalence rate in community-based samples. ${ }^{3}$ Conducting studies in such a population could lead to selection bias. By nature, individuals from such samples are more likely to have more severe and complex forms of the disorder. ${ }^{4}$ This might explain the high prevalence rate of combined ASPD/SSPD observed in the study. It would have been more interesting to know the number of individuals from this sample who have comorbidity between SSPD and other personality disorders such as narcissistic, histrionic and borderline.

Second, in the psychophysiological and behavioural characteristics of the four study groups I noticed a pattern: the comorbid ASPD/SSPD groups appear at the severe end of the spectrum, where abnormal characteristics are more frequently observed (four out of six characteristics studied in this research, namely self-reported crime and skin conductance frequency, amplitude and arousal) than the control group, which lies at the other end of the spectrum. Individuals in the ASPD and SSPD groups lie on either side of the middle of the spectrum.

A basic structural abnormality in the frontal cortex might cause these individuals to have higher psychiatric comorbidity (not only of ASPD and SSPD) and the abnormal characteristics identified might not be entirely explained by the co-occurrence of these two disorders. The authors have rightly identified that the results could not merely be due to the additive effects of both disorders, but that the increased prevalence of personality disorder in the study population might be due to a common confounder that does not lie on the causal pathway between ASPD/SSPD and abnormality in characteristics.

1 Schug RA, Raine A, Wilcox RR. Psychophysiological and behavioural characteristics of individuals comorbid for antisocial personality disorder and schizophrenia-spectrum personality disorder. Br J Psychiatry 2007; 191 408-14

2 De Girolamo G, Dotto P. Epidemiology of personality disorders. In New oxford Textbook of Psychiatry (eds MG Gelder, JJ Lopez-Ibor, NC Andreasen) 959-64. Oxford University Press, 2000.

3 Samuels J, Eaton WW, Bienvenu OJ 3rd, Brown CH, Costa PT Jr, Nestadt G. Prevalence and correlates of personality disorder in a community sample. $B$ J Psychiatry 2002; 180: 536-42.

4 Ajetunmobi O. Making Sense of Critical Appraisal. Hodder Arnold, 2002.

Murali K Sekar, Derbyshire Mental Health Services NHS Trust, Derby, UK. Email: drmurali98@yahoo.com; Ramanathan Ganapathy, Birmingham and Solihull Menta Health NHS Trust, Birmingham, UK

doi: 10.1192/bjp.192.3.233

Authors' reply: We are grateful for thoughtful critical points raised by Drs Sekar and Ganapathy. We agree that the prevalence of personality disorders in our sample is high. This sample was chosen for its elevated rates of ASPD - perhaps attributable to a downward drift in occupational functioning due to antisocial features. It is certainly vulnerable to selection bias for several reasons (among them being the fact that some individuals did not meet inclusion criteria), and the $52.4 \%$ personality disorder prevalence rate should not be mistaken for a typical community population base rate, or a general rate applying to other populations (claims which were not made in our paper). Also, the prevalence rates of other individual personality disorders (e.g. narcissistic personality disorder - around 3\%) were consistent with general population estimates. ${ }^{1,2}$ Additionally, our focus was the characteristics of this ASPD/SSPD group, rather than making any assertions about its prevalence in the general population.

On reading the correspondents' comment about SSPD comorbidity with other personality disorders, we too became intrigued with this possibility and have since conducted further analyses. These revealed, among the other personality disorders, significant SSPD comorbidity only with narcissistic personality disorder. In fact, all three of our sample's individuals with narcissistic personality disorder had comorbid ASPD and SSPD. This additional comorbidity among our ASPD/SSPD group is not surprising, given the problematically high overlap of narcissistic personality disorder with ASPD and other Cluster B disorders, ${ }^{2,3}$ the conceptual link between the narcissistic and antisocial personalities (e.g. Kernberg's 'malignant narcissism'), ${ }^{3}$ and the positive correlations observed between narcissistic personality disorder and other antisocial personalities such as psychopathy. ${ }^{4}$ We still believe ASPD/SSPD comorbidity to be meaningful, and not an artifact of the sample, as $50 \%$ of individuals with comorbid ASPD/SSPD were not characterised by any additional Axis II comorbidity.

We agree that the frontal cortex (specifically the prefrontal cortex) may be a common abnormality and that this needs further investigation. Clearly, additional research is needed on this comorbid group, in both clinical and nonclinical populations including 'unique' community samples such as our own.

1 American Psychiatric Association. Diagnostic and Statistical Manual of Mental Disorders, Text Revision (4th edn, DSM-IV-TR). APA, 2000.

2 Levy KN, Reynoso JS, Wasserman RH, Clarkin JF. Narcissistic personality disorder. In Personality Disorders: Toward the DSM-V (eds W O'Donohue, KA Fowler, SO Lilienfeld): 233-77. Sage Publications, 2007.

3 Millon T, Davis RD. Disorders of Personality: DSM-IV and Beyond. John Wiley \& Sons, 1996

4 Hare RD. Hare Psychopathy Checklist Revised (PCL-R) (2nd edn). Multi-Health Systems, 2003.

Robert A. Schug, Department of Psychology, University of Southern California, Lo Angeles, California, USA. Email: schug@usc.edu; Adrian Raine, Rand R. Wilcox, Department of Psychology, University of Southern California, Los Angeles, California, USA

doi: 10.1192/bjp.192.3.233a

\section{Patient choice in psychiatry in low- and middle- income countries}

Samele et al ${ }^{1}$ have highlighted the implications of patient choice in psychiatry and some of its main challenges. The importance of a patient-oriented approach in psychiatry has even been emphasised in the World Health Report. ${ }^{2}$ Patients seem to want more say in their treatment decisions, to receive appropriate information on their condition and make decisions concerning the management of their illness. ${ }^{3}$ Psychiatry is a particularly challenging area with regard to this, because mental illness can affect both understanding and decision-making abilities. This topic has significance particularly to low- and middle-income countries such as Sri Lanka. The attitudes of patients and choice of therapy in low- and middle-income countries may differ from those in high-income countries as cultural norms and beliefs play a major role in decision-making. ${ }^{4}$ Also, almost all the time, patients depend on the therapist to make decisions regarding their treatment either because they themselves are not knowledgeable 
enough or they think the therapist knows best. At present, suing by patients is found less frequently in low- and middle-income countries compared with the West, hence therapists are not under pressure when making decisions. This may contribute to the maintenance of the 'therapist-centred' approach in the management of patients in our part of the world. Another reason which makes patient choice less feasible in low- and middle-income countries is the limited number of therapeutic options, owing to lack of resources. This sometimes leads to medications being the only available option although other treatment modalities are indicated for the particular condition. Another factor which might impede patient choice is the lack of a proper mental health act. Some low- and middle-income countries either do not have a mental health act $^{2}$ or the existing mental health acts are archaic, were developed during the pre-antipsychotic era and are not at all patient centred. Psychiatrists and policy makers, particularly in low- and middle-income countries, should be aware of these important issues when health plans are made and implemented.

1 Samele C, Lawton-Smith S, Warner L, Mariathasan J. Patient choice in psychiatry. Br J Psychiatry 2007; 191: 1-2.

2 World Health Organization. The World Health Report 2001. Mental Health: New Understanding, New Hope. World Health Organization, 2001.

3 Laugharne R. Psychiatry in the future. The next 15 years: postmodern challenges and opportunities for psychiatry. Psychiatr Bull 2004; 28: 317-18.

4 Soltani A, Moayyeri A, Raza M. Impediments to implementing evidence-based mental health in developing countries. Evid Based Ment Health 2004; 7: 64-6.

K. A. L. A. Kuruppuarachchi, Faculty of Medicine, University of Kelaniya, Ragama, Sri Lanka. Email: lalithkuruppu@lycos.com; Aruni Hapangama, Faculty of Medicine, University of Kelaniya, Ragama, Sri Lanka

doi: 10.1192/bjp.192.3.233b

\section{Corrections}

Hypothesis: social defeat is a risk factor for schizophrenia? BJP, 191 (suppl 51), s9-s12. The axes of the graph (Fig. 1, page s11) should be labelled $x$-axis: Dopamine release; $y$-axis: $N$ (number of individuals), as shown below.

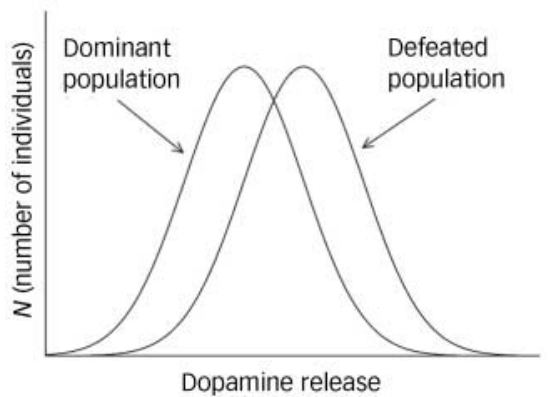

Metabotropic glutamate receptor agonists for schizophrenia. BJP, 192, 86-87. The doi for this paper is 10.1192/bjp.bp.107.045088 (corrected online, in deviation from print).

I Am by John Clare. BJP, 192, 149. The doi for this item is 10.1192/bjp.192.2.149 (corrected online, in deviation from print). 